\title{
BRCA2 mutation and Hypopharyngeal Squamous Cell Carcinoma
}

\author{
Tatiana Correa ${ }^{1}$, Douglas Laux ${ }^{1}$, and Henry Hoffman ${ }^{2}$ \\ ${ }^{1}$ University of Iowa Hospitals and Clinics \\ ${ }^{2}$ Univ. of Iowa Hospitals and Clinics
}

May 5, 2020

\begin{abstract}
Hypopharyngeal squamous cell carcinoma (SCC) has the highest mortality of all head and neck SCC subsites and typically presents in patients with history of tobacco and alcohol use. We present a case of hypopharyngeal SCC in a patient with no traditional risk-factors and a germline BRCA2 mutation.
\end{abstract}

\section{Introduction}

Head and neck squamous cell carcinomas (HNSCCs) are prevalent and have high mortality rates that have remained largely unchanged in the last decade. In contrast, outcomes for many cancers have improved as a result of increased molecular understanding and personalized management. The persistent high mortality and clinical heterogeneity of HNSCC supports the need for a similar analysis.

Hypopharyngeal squamous cell carcinoma (SCC) has the poorest prognosis of all HNSCC subsites (oral cavity, oropharynx, larynx) thought in-part due to detection at late stages. Over two-thirds of patients with a hypopharyngeal SCC present with stage IV disease. ${ }^{1}$ Five-year survival is much worse for stage IV $(22 \%)$ compared to stage I (63\%) andf stage II (57\%). Early detection of hypopharyngeal tumors is challenging due to the less accessible location and the nonspecific nature of initial symptoms, which often mimic common benign disorders such as laryngopharyngeal reflux.

Analysis of risk factors for cancer may direct more comprehensive evaluation for patients with early stage hypopharyngeal SCC. Known risk factors for hypopharyngeal SCC include tobacco use, heavy alcohol use, Plummer-Vinson Syndrome-associated iron deficiency anemia and Fanconi Anemia. ${ }^{1-2}$ To our knowledge, germline BRCA2mutations have not been previously identified as a risk factor for hypopharyngeal SCC and awareness previously-suggested associations with SCC at other head and neck subsites is limited. We present a case of hypopharyngeal SCC with a concurrent germline BRCA2 mutation and bring attention to potential diagnostic and management implications.

\section{Case Report}

An 80-year-old healthy male with no history of tobacco or alcohol use presented to an otolaryngologist for throat pain, fever, right otalgia and right maxillary sinus tenderness. He had previously been identified with a germline BRCA2 mutation in the course of successful management of intraductal breast carcinoma, prostatic adenocarcinoma and pancreatic adenocarcinoma. His initial physical exam was normal, and he was empirically treated for bacterial sinusitis. On follow up, the patient had improvement of initial symptoms but had developed a non-productive cough. His exam at that time was remarkable for a small pharyngeal ulcer, and anti-gastroesophageal reflux therapy was started.

Subsequently the patient underwent CT larynx without contrast by his Oncologist for additional workup of fatigue in the context of ongoing otalgia, sinus tenderness and cough. This imaging revealed an asymmetric 
soft tissue prominence at the larynx with thickening along the adjacent right hypopharynx, a $1.3 \mathrm{~cm}$ lymph node and fat stranding at the right carotid space. Laryngopharyngitis was diagnosed based on repeat exam and review of imaging. A primary care physician then obtained a non-contrast MRI for new headaches incidentally identifying a transglottic mass invading thyroid cartilage with encasement of the right carotid, and a $1.6 \mathrm{~cm}$ right level IIA lymph node (figure 1). The patient was referred to our tertiary care center for concern of hypopharyngeal malignancy.

Evaluation with flexible fiberoptic laryngoscopy revealed a mass at the right pharyngeal wall extending to the right pyriform sinus (figure 2). Ultrasound-guided needle biopsy was attempted twice revealing acute inflammation and keratinized squamous cell epithelium of the right piriform sinus, respectively. Biopsy of the right level II lymph node showed chronic lymphocytic leukemia. A biopsy of the right piriform sinus under general anesthesia was then completed revealing well-differentiated keratinizing squamous cell carcinoma.

A diagnostic PET/CT showed an FDG-avid soft tissue mass centered on the right piriform sinus with extension into the right parapharyngeal soft tissues, thyroid cartilage, and prevertebral space to the level of the cricoid cartilage. Chemoradiation (using weekly carboplatin and paclitaxel) was successfully completed for the stage IVa (cT4b, cN0, M0) disease. A twelve-week post-chemoradiation PET/CT revealed complete resolution of cancer and the patient remained disease-free at most recent 3-year follow up.

\section{Discussion}

Hypopharyngeal SCCs account for $<10 \%$ of HNSCCs and are difficult to visualize at early stages. ${ }^{1}$ Patients diagnosed with hypopharyngeal SCCs are predominantly males in their early 60s with a history of smoking and heavy alcohol use. These risk factors are a key determinant of clinical suspicion since tumors are associated with non-specific early symptoms.

BRCA2 mutations in HNSCC have been reported in the context of genetic variants in some patients with Fanconi Anemia, which is associated with hypopharyngeal SCC. These patients are typically young adult females with history of hematologic malignancies who develop HNSCCs in their third and fourth decade. ${ }^{2}$ One other truncating BRCA2 mutation has been linked to hypopharyngeal SCC, however, lung SCC is the most common with this mutation and breast cancer is rare. ${ }^{3}$ In some studies of families with a BRCA2 mutation, a significant increased risk of laryngeal and pharyngeal cancer has been identified. ${ }^{3}$

BRCA2 mutations can result in many cancer phenotypes due to the vital role of the BRCA2 protein in DNA homologous recombination repair. The BRCA2 gene is involved in the Fanconi Anemia (FA)/BRCA pathway for double-strand DNA break repair, which has been implicated in tumor development for both sporadic and Fanconi Anemia-related HNSCC. ${ }^{3-4}$ In this pathway, BRCA2 and other FA/BRCA proteins form a complex for DNA cross-link repair through homologous recombination (HR). Mutations at genes involved in the HR complex can impair DNA repair and potentiate cancer formation.

BRCA2 mutations may have management implications by enhancing platinum-based chemotherapy response in cancer cells from deficient DNA repair leading to increased platinum-DNA adducts. ${ }^{4}$ This interaction could explain the excellent chemoradiation response observed in our patient and has been supported for HNSCCs previouslyin vitro ${ }^{4}$ In other malignancies including ovarian, prostate and pancreatic cancers, germline BRCA2 mutations have been associated with clinically distinct tumor phenotypes that respond better to agents targeting the FA/BRCA HR pathway. ${ }^{5}$ Genetic screening to direct use of platinum-based therapies, immunotherapy and PARP inhibitors is currently underway for these malignancies suggesting similar targeted therapy potential in HNSCC.

\section{Conclusion}

This case report draws attention to a potential link between germline BRCA2 mutations and hypopharyngeal cancer. Patients with germline $B R C A 2$ mutations may benefit from more intensive workup when exhibiting symptoms commonly attributed to laryngopharyngitis. Successful treatment with conventional chemoradiation identifies potential increased chemo-radio-sensitivity of hypopharyngeal cancer associated with BRCA2 mutations. 


\section{Author Contribution}

TC wrote the manuscript, conducted literature review and synthesized correlations with Fanconi Anemia and targeted cancer therapies. DEL provided oversight of oncologic interpretations and critically revised the manuscript. HTH contributed the presented case, design of the manuscript and critically revised the manuscript. All authors reviewed and approved the final version of the manuscript.

\section{References}

1. Eckel HE, Bradley PJ. Natural History of Treated and Untreated Hypopharyngeal Cancer. Adv Otorhinolaryngol . 2019; 27-34. doi:10.1159/000492305

2. Chandrasekharappa SC, Chinn SB, Donovan FX, et al. Assessing the spectrum of germline variation in Fanconi anemia genes among patients with head and neck carcinoma before age 50. Cancer . 2017;123(20):3943-3954. doi:10.1002/cncr.30802

3. Delahaye-Sourdeix M, Anantharaman D, Timofeeva MN, et al. A Rare Truncating BRCA2 Variant and Genetic Susceptibility to Upper Aerodigestive Tract Cancer. J Natl Cancer Inst . 2015;107(5). doi:10.1093/jnci/djv037

4. Kemp SRM-D, Brink A, Meulen IHVD, et al. The FA/BRCA Pathway Identified as the Major Predictor of Cisplatin Response in Head and Neck Cancer by Functional Genomics. Mol Cancer Ther . 2016;16(3):540550. doi:10.1158/1535-7163.mct-16-0457

5. Wattenberg, M.M., Asch, D., Yu, S. et al. Platinum response characteristics of patients with pancreatic ductal adenocarcinoma and a germline BRCA1, BRCA2 or PALB2 mutation. Br J Cancer 122, 333-339 (2020). https://doi.org/10.1038/s41416-019-0582-7

Figure 1: T2 weighted axial MRI image of tumor invading right thyroid cartilage and encasing right carotid.

Figure 2: Right piriform sinus mass extending to right pharyngeal wall with minimal medial disruption in appearance of glottic and supraglottic structures.






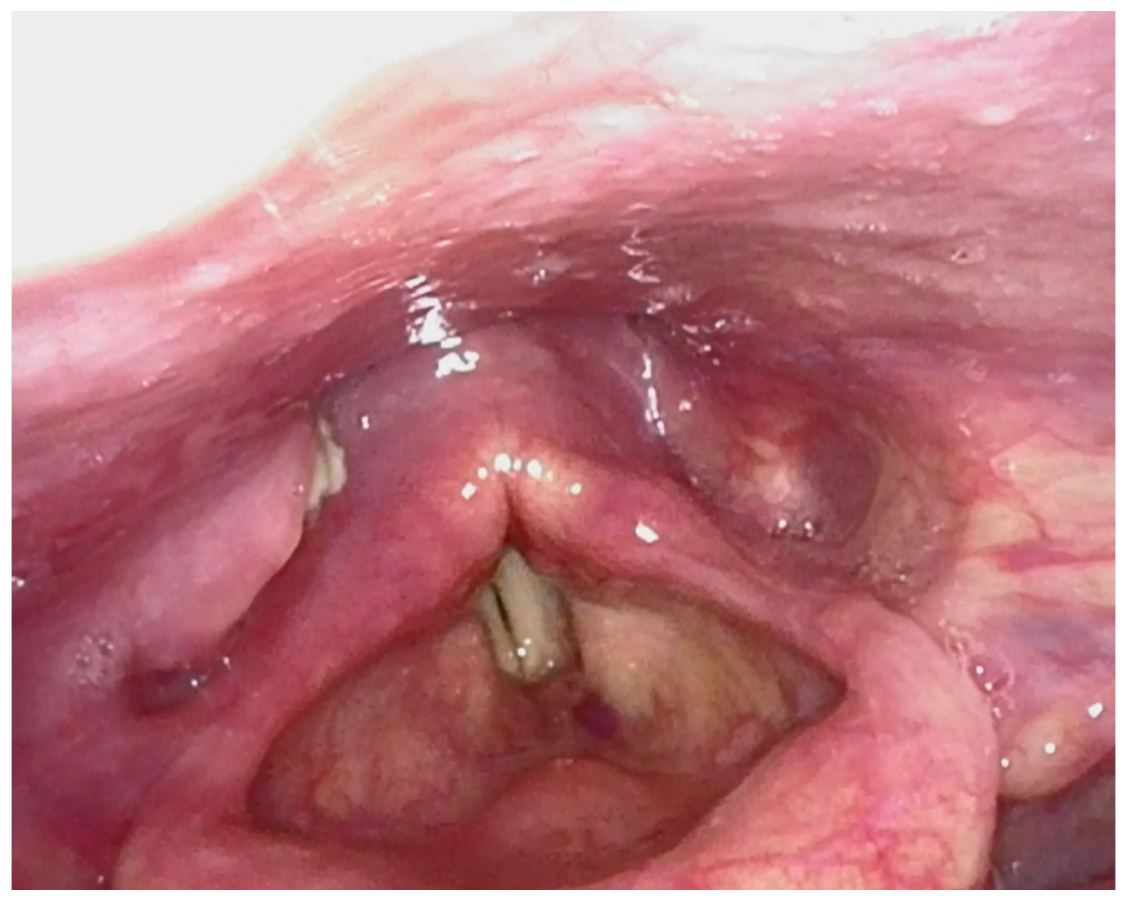

